\title{
USE OF MELDONIUM IN THE TREATMENT OF PATIENTS WITH CORONARY ARTERY DISEASE AND CONCOMITANT ARTERIAL HYPERTENSION
}

\author{
Denys Volynskyil \\ denys_volynskyi@ukr.net \\ Ihor Vakaliuk ${ }^{1}$ \\ vakaluk@gmail.com \\ ${ }^{1}$ Department of Internal medicine No. 2 and nursing \\ Ivano-Frankivsk National Medical University \\ 2 Galytska str., Ivano-Frankivsk, Ukraine, 76018
}

\begin{abstract}
Coronary artery disease (CAD) remains one of the leading causes of mortality and disability in Ukraine. Arterial hypertension (AH) is one of the most common diseases and a leading risk factor for coronary artery disease.

The aim of the work is to evaluate the antianginal activity of meldonium in the complex therapy in patients with CAD with stable angina and concomitant $\mathrm{AH}$.

Materials and methods. The study included 82 patients with CAD, stable angina pectoris II-III functional class, including 52 patients with concomitant AH stage II. The patients were divided into 2 groups. Patients in group 1 were prescribed meldonium at a dose of $750 \mathrm{mg} / \mathrm{d}$ for 2 months in addition to basic therapy for the underlying disease. Patients in group 2 continued basic antianginal, disaggregant, hypolipidemic therapy.

Results. The use of meldonium led to a decrease in the frequency of angina attacks and the need for nitroglycerin. From the 1st month of therapy and up to 2 months treatment decreased it consumption by 63 and $82.3 \%$ respectively. Adding meldonium to basic therapy led to a likely reduction in shortness of breath, episodes of palpitations, tinnitus, and headache. In all patients, after the treatment, an increase in exercise tolerance was observed, which was more pronounced in the group where patients were receiving meldonium. In the group of patients receiving meldonium, normalisation of blood pressure was faster and more pronounced.

Conclusions. Meldonium has antianginal activity, which is manifested by an increase in the physical tolerance of patients, a decrease in the frequency of angina attacks, the need for sublingual nitroglycerin intake and improvement in the well-being of patients. Additional use of meldonium promotes faster and better normalization of blood pressure. The use of meldonium in the complex therapy of patients with stable angina and concomitant $\mathrm{AH}$ allows to increase the effectiveness of traditional antianginal therapy and to improve the quality of life of such patients.
\end{abstract}

Keywords: meldonium, coronary artery disease, arterial hypertension, antianginal activity, cytoprotectors.

DOI: $10.21303 / 2504-5679.2019 .001018$

\section{Introduction}

Coronary artery disease (CAD) remains one of the leading causes of mortality and disability in Ukraine and abroad [1, 2]. Moreover, the prevalence of stable angina and heart failure in Eastern Europe patients in comparing with other developed countries was observed (4.0 and 7.8 times respectively) [3]. Arterial hypertension (AH) is one of the most common diseases and a leading risk factor for coronary artery disease, including myocardial infarction, stroke, vascular lesions, chronic kidney disease, and premature death $[4,5]$.

The treatment of coronary artery disease often remains a difficult task, due to the limited capabilities of antianginal drugs and the presence of a number of contraindications for cardiac surgery. This is especially true for patients with concomitant hypertension, which significantly worsens the patient's condition and increases the risk of complications. One of the promising directions for improving the therapy of coronary heart disease is the use of myocardial cytoprotectors included in the complex therapy. The pharmacokinetics of modern cytoprotectors are based on their ability to increase myocardial resistance to ischemia, to maintain the viability of ischemic tissues and to restore their functional activity [6]. In addition, they have pleiotropic effects. One of them is 
the stimulation of endothelial nitric oxide (NO) synthesis, which provides natural vasodilation and helps to reduce blood pressure $[7,8]$.

The key pathogenic link in the development of coronary artery disease is ischemia, as well as the activation of free radical processes in ischemic tissue, which destroy cellular structures. When concomitant hypertension is present, tissue deterioration is due to endothelial dysfunction on the background of increased blood pressure (BP). That is why the use of cytoprotectors, which have antioxidant properties and can also positively affect NO excretion, is appropriate [9, 10].

The aim of the work was to evaluate the antianginal activity of meldonium in the complex therapy of patients with coronary artery disease with stable angina and concomitant arterial hypertension.

\section{Materials and methods}

The study included 82 patients with coronary artery disease, stable angina II (43 people) III (39 people) functional classes $(53.3 \pm 7.5$ years, men -71 , women -11$)$, of whom -52 patients with concomitant hypertension (hypertension) stage II. All of them were examined and observed on the basis of the myocardial infarction department No. 2 of the Ivano-Frankivsk regional clinical cardiology centre from 2017 to 2019.

Inclusion criteria: age 40-60 years; the presence of coronary artery disease, stable angina pectoris II-III functional class, confirmed clinically (typical chest pain syndrome) and with paired veloergometry (VEM) - depression of the segment ST $>1 \mathrm{~mm}$; previous intake of cardioselective $\beta$-blockers (bisoprolol or metoprolol) and/or prolonged nitrates (isosorbide-5-mononitrate), acetylsalicylic acid, atorvastatin or rosuvastatin in individual dose regimens before inclusion into research for 4 months at least; the presence of informed consent.

Exclusion criteria: individual drug intolerance; hemodynamically significant disorders of rhythm and conduction; history of acute coronary syndrome earlier than 6 months before the start of the research; AH III stage; chronic circulatory insufficiency greater than stage IIA and greater than II FC; obesity of any genesis more than II degree; diabetes mellitus or impaired glucose tolerance; chronic renal, hepatic and respiratory failure.

The members of the Ethics Commission (extract from protocol No. 8 dated January 23, 2017) at the Ivano-Frankivsk National Medical University decided that this study would not contradict the main provisions of the GCP, Convention Council of Europe on human rights and biomedicine, the Helsinki Declaration of the World Medical Association on ethical principles for the conduct of scientific medical research with the participation of man and the Law of Ukraine "On Medicines". All patients signed an informed consent to participate in a clinical trial.

Patients were divided into 2 groups of 42 and 40 patients, respectively. For patients in group 1 meldonium were prescribed at a dose of $750 \mathrm{mg} / \mathrm{d}$ for 2 months in addition to basic therapy for the underlying disease. Patients in group 2 continued the basic antianginal, disaggregant, hypolipidemic therapy. For each patient from group 2 first a dose of $\beta$-blocker was selected, and only in the case of its inefficiency, prolonged nitrates (isosorbide-5-mononitrate) were administered. Patients' tolerance to physical activity was assessed by step intermittent paired VEM tests (duration of each stage and pause was $4 \mathrm{~min}$ ) with the calculation of threshold power $\left(\mathrm{W}_{\text {threshold }}\right)$, as well as the total power $\mathrm{W}_{\text {total }}=\left(\mathrm{W}_{1}+\mathrm{W}_{2}+\ldots\right) * 4+\mathrm{W}_{1 \mathrm{~s}} * \mathrm{~T}_{1 \mathrm{~s}} / 60$, where $\mathrm{W}_{1}, \mathrm{~W}_{2}$ is the power of fully completed stages, $\mathrm{T}_{1 \mathrm{~s}}$ is the duration of the last stage (min), the double execution index $\left(\mathrm{DEI}=\mathrm{W}_{\text {threshold }}, \mathrm{SBP} / 100\right.$, where $\mathrm{SBP}$ is a systolic blood pressure at the height of the threshold load). The total load duration $\left(\mathrm{T}_{\text {total }}\right)$ was recorded. Additionally, a 6-minute walk test was performed. The need for sublingual nitroglycerin and the frequency of angina attacks per day were evaluated. The dynamics of indicators of systolic and diastolic blood pressure (SBP and DBP, respectively) were evaluated. The dynamics of additional clinical features of patients such as shortness of breath, palpitations, rapid fatigue, heart failure, tinnitus, headache and dizziness were evaluated. All investigated parameters were evaluated monthly throughout the observation period.

Statistical treatment of the obtained results was carried out with the program "Statistica 6.0" (Free Trial Version https://www.tibco.com/), using the Newman-Keuls criterion, the data were considered reliable when the difference between the mean values $\mathrm{p}<0.05$. 


\section{Results}

The use of meldonium led to a decrease in the frequency of angina attacks and the need for nitroglycerin. From the $1^{\text {st }}$ and up to 2 months of treatment, it decreased by 63 and $82.3 \%$ respectively (Table 1). There was no significant change in these parameters during basic therapy.

Table 1

Effect of meldonium therapy on the frequency of angina attacks and the daily need for nitroglycerin in doses $(1 \mathrm{dose}=0.5 \mathrm{mg})$ in patients with coronary heart disease

\begin{tabular}{|c|c|c|c|c|}
\hline \multirow{2}{*}{ Indicator } & \multirow{2}{*}{ Group } & \multirow{2}{*}{ Before treatment } & \multicolumn{2}{|c|}{ Treatment period } \\
\hline & & & 1 month & 2 month \\
\hline \multirow{2}{*}{ Attacks per day } & meldonium & $3.11 \pm 0.24$ & $1.15 \pm 0.33^{*}$ & $0.55 \pm 0.16^{*}$ \\
\hline & basic therapy & $2.11 \pm 0.34$ & $1.76 \pm 0.33$ & $1.83 \pm 0.34$ \\
\hline \multirow{2}{*}{$\begin{array}{l}\text { Nitroglycerin per day, } \\
\text { number of doses }\end{array}$} & meldonium & $2.15 \pm 0.42$ & $1.3 \pm 0.48^{*}$ & $0.69 \pm 0.25^{*}$ \\
\hline & basic therapy & $2.25 \pm 0.22$ & $1.92 \pm 0.33$ & $1.85 \pm 0.25$ \\
\hline
\end{tabular}

Note: ${ }^{*} p<0.05$ - significance of differences with the original data

When assessing the physical tolerance of patients taking meldonium, it was found that the value of $\mathrm{W}_{\text {threshold }}$ after 1 month treatment increased by $24.7 \%$, after 2 month - by $26.1 \%$. $\mathrm{W}_{\text {total }}$ value also increased after 1 month by $50 \%$, after 2 months - by $58.6 \%$. At the same time, $\mathrm{T}_{\text {total }}$ and DEI increased (Table 2).

Table 2

Dynamics of VEM test indices in patients with coronary artery disease with meldonium treatment

\begin{tabular}{|c|c|c|c|c|}
\hline \multirow{2}{*}{ Indicator } & \multirow{2}{*}{ Group } & \multirow{2}{*}{ Before treatment } & \multicolumn{2}{|c|}{ Treatment period } \\
\hline & & & 1 month & 2 month \\
\hline \multirow{2}{*}{$\mathrm{W}_{\text {threshold }}, \mathrm{W}$} & meldonium & $67.54 \pm 3.74$ & $84.23 \pm 4.32 *$ & $85.14 \pm 4.35^{*}$ \\
\hline & basic therapy & $74.77 \pm 4.23$ & $72.14 \pm 4.55$ & $78.33 \pm 4.94$ \\
\hline \multirow{2}{*}{$\mathrm{W}_{\text {total }}, \mathrm{W}$} & meldonium & $461.6 \pm 24.6$ & $692.4 \pm 31.1^{*}$ & $732.2 \pm 25.1^{*}$ \\
\hline & basic therapy & $445.6 \pm 23.6$ & $451.4 \pm 26.1$ & $435.1 \pm 25.1$ \\
\hline \multirow{2}{*}{$\mathrm{T}_{\text {total }}, \min$} & meldonium & $6.6 \pm 3.3$ & $9.3 \pm 3.5^{*}$ & $9.3 \pm 3.9^{*}$ \\
\hline & basic therapy & $7.3 \pm 3.2$ & $7.6 \pm 3.3$ & $7.1 \pm 2.6$ \\
\hline \multirow{2}{*}{ DEI, CU } & meldonium & $182.5 \pm 6.2$ & $198.5 \pm 5.2^{*}$ & $205.3 \pm 6.1^{*}$ \\
\hline & basic therapy & $175.3 \pm 6.5$ & $174.6 \pm 5.4^{*}$ & $182.3 \pm 5.1$ \\
\hline
\end{tabular}

Note: $* p<0.05$ - significance of differences with the original data

In all patients with coronary artery disease, which were included in the study, a positive trend in reducing the frequency of detection of subjective clinical signs of the disease was observed.

Adding meldonium to basic therapy led to a likely reduction in shortness of breath, episodes of palpitations, tinnitus, and headache. However, it should be noted that in patients with coronary heart disease there was no significant reduction in rapid fatigue and dizziness (Table 3 ).

In all patients, after treatment, an increase in exercise tolerance was observed, which was more pronounced in the group where patients were receiving meldonium, as evidenced by the results of a 6-minute walk (Table 4).

All patients showed a decrease in SBP and DBP levels after treatment. In the group of patients receiving meldonium, normalisation of blood pressure was faster and more pronounced (Table 5). 
Table 3

Dynamics of clinical signs in patients with coronary heart disease with meldonium treatment (absolute data provided)

\begin{tabular}{|c|c|c|c|c|}
\hline \multirow{2}{*}{ Indicator } & \multirow{2}{*}{ Group } & \multirow{2}{*}{ Before treatment } & \multicolumn{2}{|c|}{ Treatment period } \\
\hline & & & 1 month & 2 month \\
\hline \multirow{2}{*}{ Dyspnea } & meldonium & $28(66.7 \%)$ & $25(59.5 \%)^{*}$ & $22(52.4 \%)^{*}$ \\
\hline & basic therapy & $29(72.5 \%)$ & $28(70 \%)$ & $26(65 \%)$ \\
\hline \multirow{2}{*}{ Palpitation } & meldonium & $24(57.1 \%)$ & $21(50 \%)^{*}$ & $19(45.2 \%)^{*}$ \\
\hline & basic therapy & $18(45 \%)$ & $18(45 \%)^{*}$ & $16(40 \%)^{*}$ \\
\hline \multirow{2}{*}{ Rapid fatigue } & meldonium & $36(85.7 \%)$ & $27(64.3 \%)$ & $25(59.5 \%)$ \\
\hline & basic therapy & $34(85 \%)$ & $30(75 \%)$ & $30(75 \%)$ \\
\hline \multirow{2}{*}{$\begin{array}{l}\text { Interruptions in the } \\
\text { heart activity }\end{array}$} & meldonium & $16(38.1 \%)$ & $15(35.7 \%)^{*}$ & $15(35.7 \%)^{*}$ \\
\hline & basic therapy & $17(42.5 \%)$ & $16(40 \%)$ & $15(37.5 \%)$ \\
\hline \multirow{2}{*}{ Tinnitus } & meldonium & $23(54.8 \%)$ & $22(52.4 \%)^{*}$ & $19(45.2 \%)^{*}$ \\
\hline & basic therapy & $12(30 \%)$ & $11(27.5 \%)^{*}$ & $9(22.5 \%)^{*}$ \\
\hline \multirow{2}{*}{ Headache } & meldonium & $29(69 \%)$ & $24(57.1 \%)^{*}$ & $20(47.6 \%)^{*}$ \\
\hline & basic therapy & $32(80 \%)$ & $30(75 \%)$ & $29(72.5 \%)$ \\
\hline \multirow{2}{*}{ Dizziness } & meldonium & $21(50 \%)$ & $18(42.9 \%)$ & $14(33.3 \%)$ \\
\hline & basic therapy & $24(60 \%)$ & $22(55 \%)$ & $21(52.5 \%)$ \\
\hline
\end{tabular}

Note: $*-p<0.05$ - significance of differences with the original data

Table 4

Results of a 6-minute walk test in patients with coronary artery disease on the background of receiving meldonium

\begin{tabular}{cccc}
\hline Indicator & Group & Before treatment & After treatment \\
\hline \multirow{2}{*}{ 6 minute walk distance, $\mathrm{m}$} & meldonium & $362.8 \pm 23.7$ & $507.3 \pm 22.1^{*}$ \\
& basic therapy & $353 \pm 20.8$ & $482 \pm 24.5^{*}$
\end{tabular}

Note: ${ }^{*}-p<0.05$ - significance of differences with the original data

Table 5

Dynamics of blood pressure in patients with coronary heart disease with concomitant hypertension on the background of treatment with meldonium

\begin{tabular}{|c|c|c|c|}
\hline Indicator & Group & Before treatment & After treatment \\
\hline \multirow{2}{*}{ SBP mmHg } & meldonium & $152.4 \pm 8.9$ & $129.6 \pm 3.56^{*}$ \\
\hline & basic therapy & $155.6 \pm 9.6$ & $138 \pm 4.87^{*}$ \\
\hline \multirow{2}{*}{ DBP mmHg } & meldonium & $93.8 \pm 6.37$ & $79.63 \pm 6.12^{*}$ \\
\hline & basic therapy & $94.5 \pm 5.3$ & $81.36 \pm 4.08 *$ \\
\hline
\end{tabular}

Note: $*-p<0.05$ - significance of differences with the original data

\section{Discussion}

The study allowed us to confirm data on the presence of antianginal activity in meldonium. It is known that the major source of energy for cardiomyocytes is free fatty acids (FFA) and carbohydrates, in particular glucose. During the experiments, it was proved that for synthesis of the 
same number of adenosine triphosphate (ATP) molecules using glucose rather than free fatty acids, $16-30 \%$ less oxygen molecules are needed $[11,12]$. Only glucose can participate in redox reactions in cells in the complete absence of $\mathrm{O}_{2}$. In the development of ischemia for myocardial tissues, the most energy-efficient ways of synthesis of ATP molecules are glycolysis and glycogenolysis. However, the utilization of that small amount of oxygen, which still enters the ischemic zone, is ensured mainly by the oxidation of free fatty acids by carnitine [13]. During this reaction, there is an accumulation of waste products of $\beta$-oxidation of fatty acids (acylcoenzyme A and acylcarnitine) in the myocardium. These metabolic products contribute to the damage of cell membranes, increase the saturation of cardiomyocytes with calcium ions, reduce the potential action and, in sum, lead to an increase in the ischemic zone $[14,15]$.

The action of meldonium is based on intracellular inactivation of carnitine synthesis, which ensures the transport of FFA in the cell mitochondria. As a result, the flow of free FFA into the mitochondria decreases (a lot of oxygen is required for their oxidation), which in turn indirectly inhibits $\beta$-oxidation of FFA in the mitochondria, providing cytoprotection. In addition, meldonium by increasing the level of $\gamma$-buterobetaine stimulates the activity of NO synthase $[16,17]$. Therefore the use of the drug can reduce the severity of vascular endothelial dysfunction, which is inherent in coronary artery disease and hypertension [18]. The use of cardiocytoprotectors additionally prevents the occurrence of undesirable side effects after receiving high doses of antianginal drugs, in particular bradycardia and hypotension. Numerous clinical studies have demonstrated the anti-ischemic efficacy of meldonium in patients with coronary heart disease, which has contributed to improving the well-being and quality of life of patients as a whole $[19,20]$.

In this regard, it is advisable to use meldonium in patients with chronic coronary pathology and hypertension to increase the effectiveness of antianginal and antihypertensive therapy and increase the resistance of the body's antioxidant systems.

Study limitations. A limitation of the study is the fact that the data on changes in clinical signs in patients with coronary heart disease were based on their statements due to the lack of measured values and some subjectivity in this measurement, which could potentially be a source of inaccuracies. The lack of objective information cannot be completely avoided in real clinical practice. However, it was shown that information about dynamics of clinical signs in patients provided personally by themselves are suitable for use in clinical trials.

Prospects for further research. Insofar as all aspects of the effects of metabolic therapy and meldonium in particular on the course of coronary artery disease with concomitant hypertension are not known yet, this area of research needs further investigation.

\section{Conclusions}

The study showed that meldonium has an antianginal activity, which was manifested by an increase in the physical tolerance of patients receiving the drug, as well as a decrease in the frequency of angina attacks, the need for sublingual nitroglycerin intake and improving the well-being of patients.

Additional use of meldonium promotes faster and better normalization of blood pressure.

The use of meldonium in the complex therapy of patients with stable angina and concomitant arterial hypertension allows to increase the effectiveness of traditional antianginal therapy and to improve the quality of life of such patients.

\section{References}

[1] Anikeeva, T. V. (2012). Coronary artery lesions in patients with extracardial atherosclerosis. Issues of experimental and clinical medicine, 16, 21-24.

[2] Shalnova, S. A., Oganov, R. G., Deev, A. D., Imaeva, A. E., Lukyanov, M. M., Artamonova, G. V. et. al. (2015). Comorbidities of ischemic heart disease with other non-communicable diseases in adult population: age and risk factors association. Cardiovascular Therapy and Prevention, 14 (4), 44. doi: http://doi.org/10.15829/1728-8800-2015-4-44-51

[3] Shalnova, S. A., Oganov, R. G., Steg, F. G., Ford, J. (2013). Ischemic heart disease. Modern reality by data of World Register CLARIFY. Cardiology, 53, 28-33. 
[4] Gorbas, I. M. (2011). The program of response and use of arterial hypertension in Ukraine: results of research. Health of Ukraine, 3, 32-34.

[5] Gorbas, I. M., Smirnova, O. O., Kvasha, I. P., Dear, A. P. (2010). Evaluation of the effectiveness of the "Program for the prevention and treatment of hypertension in Ukraine" according to epidemiological studies. Arterial Hypertension, 6, 51-82.

[6] Mikhin, V. P., Tyurikov, P. Y. (2016). Anti-ischemic and antioxidant activity of meldonium in ihd patients with stable angina. Medical Council, 13, 56-60. doi: http://doi.org/10.21518/2079-701x-2016-13-56-60

[7] Gordeev, I. G., Lusov, V. A., Bekchiu, E. A., Volov, N. A., Ilina, E. E., Lebedeva, A. Y. (2006). Myocardial cytoprotection and ischemic myocardial dysfunction correction in stable angina patients after transluminal balloon angioplasty or coronary artery stenting. Russian Journal of Cardiology, 1, 33-39.

[8] Netyazhenko, V. Z. (2007). Treatment of stable angina in accordance with the recommendations of the European Society of Cardiologists. Changes and current provisions. Medicine and pharmacy news, 11 (217), 14-17.

[9] Mkrtchyan, V. R. (2008). Tactics of the use of means that improve the energy metabolism of the myocardium: training allowance. 20

[10] Mildronate (MET-88) - Antianginal, Cardioprotectant (2001). Drugs Fut, 26 (1), 82-86.

[11] Calvinsh, I. Y. (2002). Mildronate and trimethazidine: similarity and difference in their action. Terra medica nova, 3, 3-15.

[12] Khlebodarov, F. E., Turikov P.Y., Mikhin, V. P. (2009). Endothelial dysfunction and its correction wit cytoprotectors in patients with stable effort angina and arterial hypertension. Russian Journal of Cardiology, 6, 34-39.

[13] Vasquez Abanto, H. E. (2015). Hypertension: familiar concepts, new perspectives. Medicine and pharmacy news, 541, 11-18.

[14] Vizir, V. A., Goncharov, O. V. (2008). Activity of inflammatory processes in patients with hypertension. Ukrainian Medical Almanac, 1, 68-70.

[15] Golyachenko, O. M. (2011). Demographic processes in Ukraine in the years of independence. Bulletin of scientific research, 4, 38-41.

[16] Inglis, S., Stewart, S. (2006). Metabolic Therapeutics in Angina Pectoris: History Revisited with Perhexiline. European Journal of Cardiovascular Nursing, 5 (2), 175-184. doi: http://doi.org/10.1016/j.ejcnurse.2006.01.001

[17] Dzerve, V., Matisone, D., Kukulis, I. et. al. (2005). Mildronate improves peripheral circulation in patients with chronic heart failure: results of clinical trial (the first report). Sem. Cardiol., 11 (2), 56-64.

[18] Vilskersts, R., Liepinsh, E., Kuka, J., Cirule, H., Veveris, M., Kalvinsh, I., Dambrova, M. (2009). Myocardial Infarct Size-Limiting and Anti-Arrhythmic Effects of Mildronate Orotate in the Rat Heart. Cardiovascular Drugs and Therapy, 23 (4), $281-288$. doi: http://doi.org/10.1007/s10557-009-6179-2

[19] Neri, M., Fineschi, V., Paolo, M., Pomara, C., Riezzo, I., Turillazzi, E., Cerretani, D. (2015). Cardiac Oxidative Stress and Inflammatory Cytokines Response after Myocardial Infarction. Current Vascular Pharmacology, 13 (1), 26-36. doi: http://doi.org/ $10.2174 / 15701611113119990003$

[20] Statsenko, M. (2017). Efficacy of Short-Term Therapy With Meldonium in Patients With Chronic Heart Failure of Ischemic Etiology and Type 2 Diabetes Mellitus. Kardiologiia, 57 (4), 58-63.

Received date 21.10.2019

Accepted date 26.11.2019

Published date 30.11.2019
(C) The Author(s) 2019

This is an open access article under the CC BY license (http://creativecommons.org/licenses/by/4.0). 